\title{
LOS PERSEGUIDORES: EL JOHNNY CARTER DE JULIO CORTÁZAR EN LAS REESCRITURAS CONTEMPORÁNEAS DE ABELARDO CASTILLO, FERNANDO QUIÑONES Y ANTONIO MUÑOZ MOLINA
}

\author{
Rodrigo Guijarro Lasheras \\ Universidad Complutense de Madrid, Madrid, España \\ rguijarr@ucm.es
}

\begin{abstract}
RESUMEN/ ABSTRACT
Mediante un análisis que parte de la plasmación literaria de la figura del músico, este artículo localiza y analiza tres de las reescrituras que "El perseguidor", de Julio Cortázar, ha experimentado a lo largo de las últimas décadas. Así, se muestra en primer lugar cómo tres obras, firmadas por Abelardo Castillo, Fernando Quiñones y Antonio Muñoz Molina, constituyen una interpretación del mismo relato base. Dada esta perspectiva transatlántica, se argumenta a continuación que estas versiones suponen la fijación de una serie de invariantes que delimitan una aproximación común a la música, y que esta se encarna siempre en un personaje heredero de Johnny Carter. A su vez, llevan a cabo una reescritura que pivota en torno a la voz, el espacio y el tiempo. Esto supone enlazar con la nutrida tradición crítica y bibliográfica sobre Cortázar desde un ángulo poco frecuentado (el de la interpretación que entraña toda versión) que se antoja relevante para entender la significación y el alcance de uno de sus textos de referencia.
\end{abstract}

Palabras Clave: “El perseguidor", Julio Cortázar, reescrituras, Abelardo Castillo, Fernando Quiñones, Antonio Muñoz Molina.

THE PURSUERS:

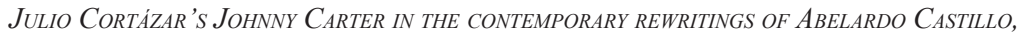
FERNANDO QUIÑONES AND ANTONIO MUÑOZ MOLINA

Through an intermedial approach that focusses on the literary depiction of the musician, this article locates and explains three rewritings that Julio Cortázar's "El perseguidor" undergone had during the last decades. Thus, three different works will be analyzed, by A. Castillo, F. 
Quiñones and A. Muñoz Molina, respectively, build up an interpretation of the short story on which they are all based. Given this transatlantic perspective, it will be argued that these versions establish a series of invariants by reinterpreting voice, time and space in the original narration. This perspective, then, joins the critical tradition and copious bibliography on the Argentinian writer from a point of view that has been seldom adopted: the interpretation that each of these versions imply. This seems to be an important issue in order to understand the meaning and scope of one of Cortázar's milestones.

KEYWords: "El perseguidor”, Julio Cortázar, rewritings, Abelardo Castillo, Fernando Quiñones, Antonio Muñoz Molina.

Recepción: 13/09/2017

Aprobación: 20/11/2017

\section{INTRODUCCIÓN}

"El perseguidor", relato publicado en 1959 y recogido en el volumen Las armas secretas, se ha convertido en uno de los textos más icónicos de Julio Cortázar. Una de las pruebas más inequívocas que en este sentido cabe aducir, más allá de los numerosos estudios críticos y ediciones existentes de la obra, es el influjo directo que ha ejercido sobre autores posteriores, que han expandido y amplificado el relato original. Esto sucede con las tres obras (dos relatos y una novela) que este artículo recopila y analiza, a saber: "Noche para el negro Griffiths" (1976) ${ }^{1}$, de Abelardo Castillo; "El testigo" $(1980)^{2}$, de Fernando Quiñones; y El invierno en Lisboa (1987), de Antonio Muñoz Molina. El objeto de este estudio es mostrar cómo la lectura de estas tres narraciones aporta una nueva visión de su hipotexto ${ }^{3}$ fundamental y contribuye a generar una visión particular de la música y del músico heredada de Cortázar. Por otro lado, cada una de ellas plantea una relectura que, como veremos, se agrupa en torno a tres ejes: temporalidad, espacio y voz. Se trata

1 Contenido en Las panteras y el templo. En sus diarios (Castillo 2014) el autor indica que lo escribió en 1964 (entrada de enero 1965), si bien más adelante señala que está incompleto. Fue un cuento, por tanto, que tardó varios años en completarse y adquirir su versión definitiva, lo cual es característico del autor. Como señala Morello-Frosch (12-14), también la reescritura de un relato preexistente, adaptándolo a un nuevo contexto actual, es un recurso habitual de Castillo.

2 Contenido en Nos han dejado solos. Libro de los andaluces.

3 Empleo el término en el mismo sentido que Genette (14), como un texto A que resuena o está presente en uno o varios textos B posteriores. Por ejemplo, las novelas de caballerías son un claro hipotexto del Quijote. 
de un caso inusual y privilegiado, ya que pocas obras contemporáneas poseen un conjunto de reescrituras tan explícitas y tan próximas en el tiempo como las que Castillo, Quiñones y Muñoz Molina proponen.

Castillo narra la historia de Israfel Sebastian Griffiths, trompetista de jazz que se gana la vida tocando en bares de escasa reputación en Barracas, barrio periférico de Buenos Aires. Ha de señalarse que, pese a la inspiración cortazariana explícita de este relato, los estudios comparativos existentes entre estos dos autores han tendido a dejarlo de lado (Aquino y Pignataro 2016 o Menczell 2002, por ejemplo). Por otro lado, el relato de Quiñones se centra en la vida de Miguel Pantalón, cantaor flamenco en el Cádiz previo a la Guerra Civil. Finalmente, Muñoz Molina confiere un destacado papel en su novela a Billy Swann, legendario trompetista americano que ofrece junto a Santiago Biralbo, personaje principal, su último y memorable concierto en Lisboa. Tanto Griffiths como el Pantalón y Billy son recreaciones de Johnny Carter ${ }^{4}$ presentadas, igual que este, a través de la mediación de un narrador personaje que entabla relación con él. La voz de estos protagonistas y su caracterización son por tanto un aspecto fundamental en las relecturas del relato cortazariano. Cada una de ellas introduce diversos matices, que aquí se analizan en los apartados dedicados a la reescritura de la voz (1 y 2), el espacio (3) y el tiempo (4), generando una visión conjunta que desemboca en las conclusiones finales (5). De entrada, no obstante, se percibe una alta cantidad de puntos comunes entre estas obras que contribuyen a delinear el perfil del músico marginal como mito literario ${ }^{5}$. Conviene comenzar, por tanto, señalando los nexos entre Johnny Parker y sus descendientes.

$4 \quad$ Es de sobra conocido que Johnny Carter es un trasunto de Charlie Parker. Para un estudio del vínculo entre el personaje real y el ficcional, cf. Borello (1980). No obstante, mi objetivo no es analizar esta relación, sino la que Johnny establece con sus alter ego posteriores.

Se trata de un músico urbano, de clase media o baja, cuyo talento no es reconocido o solo lo es por unos pocos, que con frecuencia parece más preocupado por su mundo interior que por su subsistencia y sus constantes problemas económicos. Cortázar, como me propongo mostrar, desarrolla y modula esta idea. Uno de los más claros antecedentes de esta figura es la del artista bohemio (escritor, músico, pintor) de finales del siglo XIX y principios del XX, además situado a menudo en un marco parisino. Por otro lado, la difusión de músicas populares como el jazz o el tango también dio lugar desde muy pronto a plasmaciones de un artista a partes iguales talentoso y marginal, como los que encontramos en las páginas del escritor beat Jack Kerouac o en los escritos de jazz del novelista Boris Vian. Salvando las distancias de 


\section{LA REESCRITURA DE LA VOZ: LA FORJA DE UN MITO CONTEMPORÁNEO}

El número de invariantes que pueden detectarse en los cuatro textos seleccionados es inusitadamente alto. Se trata de unas constantes que permiten observar los rasgos que definen el mito contemporáneo del músico de suburbio, cuya genialidad no está reñida con su falta de instrucción formal. Porque ni Johnny, ni Griffiths, ni el Pantalón, ni Billy poseen una educación musical propiamente dicha. "Ni música sé" (Castillo 313), afirma el segundo, refiriéndose a su incapacidad para leer partituras. Es fundamental en la construcción de este mito que todos ellos pertenezcan a un estrato socio-cultural bajo, a cuya miseria su arte no les permite escapar. "Nace en la plaza Mina y en una casa bien, y es Falla, y si Falla nace en el barrio Santa María o en La Viña, es El Pantalón" (Quiñones 478), afirma el narrador de "El testigo". Castillo, igualmente, cambia el París de Cortázar por el menos glamuroso suburbio porteño; y Quiñones, por su parte, plasma este desclasamiento en el nivel formal y lingüístico. El narrador, otro cantaor que conoció y tuvo un estrecho trato con Miguel Pantalón, se caracteriza por el reiterado uso de dialectalismos y vulgarismos ("el furbo", "el piano de Betoben", "Estocormo", "Garcías'Lorca”), así como por una divagación constante que se traduce en un monólogo pseudo-conversacional. El aspecto formal y lingüístico, por tanto, subraya el espacio social que ha de ocupar el perseguidor.

Esto conduce a otro aspecto clave presente en las cuatro obras: la inconsciencia o insatisfacción de los cuatro músicos ante su arte y genialidad. Johnny Carter llega al extremo de ordenar destruir la grabación del que el narrador considera uno de sus mejores solos (Cortázar 169). Quiñones traduce esto en el desinterés de su personaje por labrarse ningún tipo de carrera, así como por grabar su música, de la que, una vez cantada, "ni se acordaba ya ni le importaba" (Quiñones 468). El trompetista de Castillo es "consciente de sus límites como si fuera un genio" (Castillo 310) y se caracteriza también por no tener su música en alta estima. Billy Swann, por último, si bien no acusa el carácter autocrítico o inseguro de los anteriores con tanta claridad, dice ser "una sombra" de sus años de gloria, "un desterrado de aquel tiempo" (Muñoz

espacio y tiempo, algunos compositores de la tradición clásica han sido representados así en ocasiones, como le sucede a Franz Schubert en la Sinfonía inacabada de Alejandro Casona. 
Molina 116). La genialidad de los perseguidores ha de tener, por tanto, un componente de inconsciencia, de autorrechazo o, incluso, de autodestrucción.

El relato de Castillo presenta una variación de este perfil psicológico que puede ser fácilmente malinterpretada. Sería fácil afirmar que Griffiths es, sin mayor matización, un trompetista mediocre, basándose para ello en la evidencia textual explícita que ofrece el narrador al caracterizarlo justamente en estos términos. Sin embargo, el clímax del relato lo constituyen precisamente los instantes de genialidad de los que Griffiths es capaz, lo cual culmina en una prolongada descripción final de su más brillante actuación. Es una 'mediocridad', por tanto, que conviene atenuar, toda vez que supone ante todo un refuerzo del carácter dejado y arrabalero, "fracasado y canalla" (Castillo 309) del protagonista, sin excluir por ello en ningún momento su acceso al club de Johnny Carter y demás talentosos epígonos. Esto ha de enmarcarse también en el rechazo físico que el narrador siente por el músico, al que ve "como un mono" (Castillo 310), en clara deuda con el "asco infinito" hacia el aspecto de "chimpancé" (Cortázar 144) que experimenta el narrador cortazariano. Si la mediocridad no impide los instantes geniales, no es tal; si Griffiths fuera realmente mediocre, no sería una lectura (a menos que fuera paródica o desmitificadora) de Johnny Carter.

Los tres perseguidores verbalizan en algún momento (a través de la mediación de un narrador u observador, como se verá) sus ideas o sensaciones respecto de la música. Y todos ellos pronuncian lo que puede denominarse una frase intuitiva, paradójica o desconcertante, que metafóricamente condensa, a modo de eslogan, la concepción que tienen de su arte. "Esto lo estoy tocando mañana" (Cortázar 193), afirma Johnny, mostrando con ello cómo la música altera su experiencia del tiempo. Griffiths apunta igualmente que "El mundo es como círculos" (Castillo 310), mientras que Billy concluye que "No importa la maestría, sino la resonancia” (Muñoz Molina 84). Miguel Pantalón tampoco excusa esta invariante y le espeta a otro de los músicos que, mientras canta, "estoy leyendo quince libros sin saber leer" (Quiñones 471).

La frase intuitiva conduce al tema de la inefabilidad de la experiencia musical, que todas estas narraciones explotan, si bien esto halla su máxima expresión en otro rasgo invariable: el concierto epifánico. En todas las obras se narra un concierto, habitualmente el último que ofrece el protagonista, que constituye el momento culminante de su arte y ante el que el narrador queda profundamente afectado. Es la muestra de las capacidades del protagonista. "Esa cosa increíble que Johnny había soplado en Amorous" (Cortázar 170) es el punto de partida que Muñoz Molina convierte en el momento culminante 
de su novela en su penúltimo capítulo, cuando el protagonista y Billy Swann se reúnen por última vez para ofrecer en Lisboa el que será el concierto final del segundo. El narrador se recrea en la descripción de este momento y de la música de Billy, cuyas notas parecen comprender y condensar todos los temas y símbolos de la novela: Lucrecia, Lisboa, San Sebastián, el cuadro de Cézanne, la separación y ausencia de la amada, etc. Lo mismo sucede con los otros dos relatos: "Noche para el negro Griffiths" concluye con la descripción del último concierto que el narrador le escucha al protagonista, en el que, para su sorpresa, Griffiths despliega sus mejores habilidades. Esto constituye el momento climático hacia el que se dirige la narración. Finalmente, Miguel Pantalón alcanza uno de sus momentos cumbre como cantaor minutos antes de morir de improviso en el mismo local en el que ha dado su último concierto (Quiñones 479). Las tres narraciones subrayan el carácter final e involuntariamente testamentario de estas actuaciones, lo cual también se marca en el relato de Cortázar ("vaya a saber si Amorous no resulta el testamento del pobre Johnny", Cortázar 171). El hecho de que sean los últimos conciertos de los protagonistas y que se sitúen al final de la narración (lo cual no sucede en Cortázar) es una variación que, no obstante, apuntala y realza un rasgo ya presente en el texto original: su carácter epifánico y revelador.

Porque otro de los rasgos que las tres narraciones adoptan de Johnny Carter es su condición marginal. El perseguidor, según esta visión, ha de ser un outsider: sin educación, drogadicto, pobre, pero un outsider también por su extravagancia rayana en la locura. Johnny, de hecho, ha pasado temporadas internado en centros psiquiátricos. Los pasajes en los que Johnny quema la habitación de su hotel, pasa horas en el parque metiéndose hojas en los bolsillos o se muestra impúdicamente desnudo se metamorfosean en los textos elegidos en otros tantos episodios análogos. Quiñones, por ejemplo, dedica una parte considerable de su relato a la glosa de anécdotas que forman un mosaico de la personalidad de Miguel Pantalón. Miguel es un ser que desprecia o no tiene consciencia del dinero (Quiñones 469), que, como Johnny, experimenta repentinos ataques de ira y que, ante todo, se comporta de manera siempre impredecible y caprichosa. En realidad, todos estos músicos tienen como rasgo distintivo su desinterés por el mundo y su impulsividad infantil, factores que llevan a quienes los tratan a perdonar siempre sus extravagancias -Bruno lo hace en varias ocasiones, así como el narrador y demás personajes de Quiñones (472)-, con una indulgencia que se entiende mejor a la vista de esta idiosincrasia. 
El negro Griffiths, por su parte, se pierde narrando historias y pensamientos que solo él parece entender, "a tal punto que muchas veces continuaba en inglés, para él solo" (Castillo 312). Y, en El invierno en Lisboa, Billy Swann aparece siempre como un ser incapaz de adaptarse a las normas sociales establecidas y llevar una vida acomodada, estable y convencional. Por eso antes de salir al escenario por última vez, se afirma que "Billy Swann no había tocado nunca para aquella gente [el público burgués que acude a verlo] [...], porque a pesar de que estuvieran allí y hubieran comprado dócilmente sus entradas la música que iban a escuchar nunca podría conmoverlos" (Muñoz Molina 178). Los perseguidores, por tanto, son incompatibles con el estilo de vida estandarizado y las convenciones de la sociedad en la que viven y para la que tocan.

Todo ello los lleva, en último término, a la muerte. Paredes (173) la destaca como la conclusión inevitable del relato cortazariano, y así lo entienden también Quiñones y Muñoz Molina. La única excepción parece ser la de Griffiths, del que "he sabido que todavía en 1969 tocaba la trompeta por cantinas cada vez más mugrientas" (Castillo 309), y que es también el único que no cuenta entre sus rasgos con la adicción a las drogas. Sí es adicto, como luego veremos, el narrador. Salvo este caso, la muerte alcanza a los perseguidores a pesar del ángel salvador que a todos ellos les acompaña en diversos momentos de sus respectivas historias: la Dédée de Cortázar se transmuta en Cecilia, que mantiene asimismo relaciones con el narrador en el relato de Castillo, o en las monjas lisboetas de El invierno en Lisboa.

\section{LA REESCRITURA DE LA OTRA VOZ}

Lo dicho hasta ahora da cuenta de las principales invariantes que definen la figura mítica del perseguidor, esto es, los rasgos del Johnny Carter de Cortázar que sus continuadores literarios adaptan sistemáticamente a sus respectivos contextos. Esta es la voz de los perseguidores. No obstante, los tres textos (además del de Cortázar) presentan igualmente otra voz, una voz que filtra de hecho la de los protagonistas músicos y que se reelabora de distintas maneras y con diversos fines en cada una de las narraciones.

El relato de Cortázar está narrado por Bruno, crítico de jazz y amigo de Johnny. Esto se traduce, en el caso de Castillo, en un narrador en primera persona, escritor, que también tiene trato personal con el músico protagonista. La estrategia narradora de Muñoz Molina es semejante, si bien añade un 
nivel adicional: conocemos la historia de Billy a través de lo que Santiago Biralbo, también músico y auténtico protagonista de la novela, le cuenta al narrador. Este narrador, si bien homodiegético, permanece en todo momento en el anonimato y no tiene apenas incidencia sobre la trama. La figura de Billy, por tanto, está doblemente 'mediada': no solo nos llega por medio de un narrador, sino por medio de lo que un personaje le cuenta al narrador. Finalmente, Quiñones varía asimismo el planteamiento original, ya que la historia de Miguel Pantalón la conocemos a través de los recuerdos de él que guarda otro cantaor y que le cuenta a un entrevistador invisible, ya que es el cantaor quien propiamente narra la historia, si bien utiliza en alguna ocasión la segunda persona para marcar la existencia de este escritor oculto ("Ya ves: que tenías que irte-que tenías que irte y venga a apuntar, claro, es lo tuyo", Quiñones 478), que virtualmente transcribe lo que el personaje cuenta. Lo que leemos es, por tanto, una entrevista. El presente narrativo, además, está muy distanciado del tiempo de la historia, ya que el Pantalón vivió y murió antes de la Guerra Civil española, y el narrador, entonces joven, es ahora un anciano. Merece la pena señalar que, en consecuencia, el relato de Quiñones es el único en el que la voz de Miguel Pantalón no aparece reproducida directamente. No tenemos sus palabras textuales, sino que su voz está subsumida en la del colega que le ha sobrevivido. Tal vez por ello, el título ("El testigo") no hace alusión al perseguidor, sino a quien lo conoció.

Pero la cuestión de la voz narradora superpuesta a la voz del auténtico protagonista está lejos de tener un interés meramente formal. En primer lugar, el filtro a través del que nos llega la voz de los respectivos músicos es el modo idóneo para plasmar algunos de los rasgos de estos que se han ido mencionando páginas atrás: es el reflejo formal de la inconsciencia respecto de su verdadero talento - de modo que es otro quien nos lo tiene que contar-, su desajuste respecto del mundo, la sociedad y su mecánica. Además, los cambios relativos a la voz narradora apuntan ante todo a una reinterpretación de la dicotomía creador/crítico que se halla en el núcleo del relato cortazariano -véase Álvarez Borland (2005) para un mayor desarrollo de esta idea-. Quiñones y Muñoz Molina sustituyen a Bruno, crítico musical que "lo traduce todo a [s]u sucio idioma" (Cortázar 182), por sendos músicos. Esto es algo fundamental, toda vez que implica la anulación de la polaridad mencionada para establecer un diálogo entre iguales, entre dos músicos. De este modo, ambos textos implican una simplificación (puesto que se centran en la música y su intérprete, prescindiendo de la reflexión metaliteraria) a 
través de la complicación del juego de voces (puesto que, como hemos visto, incluyen un filtro narrativo adicional respecto del relato de Cortázar).

Castillo, por su parte, altera igualmente la dualidad creador/crítico, si bien no lo hace introduciendo a otro músico en el relato, sino a un escritor, también creador por tanto, que es el que narra la historia. De este modo, Castillo parece, por un lado, poner en primer plano una oposición que está presente, si bien de forma más implícita, en "El perseguidor", y que va en detrimento de la otra, fundamental, constituida por el par creador y crítico: la dualidad literatura/música. No obstante, en ningún momento entra a valorarse la obra literaria del narrador ni lo que esta supone. Más que profundizar en el contraste que pueda haber entre la obra de los dos personajes principales por el hecho de expresarse a través de medios distintos (literario y musical), lo que a Castillo le interesa es profundizar en la caracterización del antagonista. Esto es, del narrador. El narrador, de nuevo sin nombre, no sale muy bien parado: alcohólico, violento y despreciativo, establece una relación ambivalente con el músico, a quien insulta y recrimina su mediocridad sin por ello dejar de percibir los instantes sublimes que es capaz de crear con su trompeta. Esta figura, de hecho, no exenta de autocrítica, es una de las que más ha trabajado el autor argentino, protagonizando, por ejemplo, la que seguramente sea su novela más conocida, El que tiene sed.

He aquí el principal cambio de voz que existe en esta reescritura del relato: del crítico autoconsciente de sus limitaciones que lamenta la incapacidad para apresar y llegar al fondo de la genialidad de su admirado Johnny (lo que no quita para que comente la buena acogida y ventas del libro que ha escrito sobre él y pueda por momentos mostrarse condescendiente con su biografiado ${ }^{6}$ ), se pasa a un escritor que desprecia abiertamente al protagonista e incluso lo humilla. Para realzar más aún la reescritura de la voz que Castillo imprime en su narrador, son interesantes las palabras de Paredes respecto de "El perseguidor": "Aquí se explicita como tema y anécdota un aspecto esencial de la estética del autor: la escritura de un relato como una indagación de la intimidad humana, sus búsquedas vitales y lazos personales más estrechos. A través de la indisoluble amistad entre estos dos sujetos ficticios, Cortázar dice que el narrador debe ser el mejor amigo del personaje literario" (Paredes 164-165). 
El narrador de Castillo se acerca a su amigo de modo distinto a como lo hace el narrador de Cortázar. No lo biografía, no escribe sobre él, o solo lo hace de forma metaliteraria: escribe el propio relato que estamos leyendo. Pero, en cualquier caso, no tiene esa necesidad de acercamiento que implica el trabajo de Bruno respecto de Johnny. "Johnny's work is, after all, the necessary condition for Bruno's success" (Sommer 150), pero no así en el caso de Griffiths y su amigo escritor. No existe, por tanto, la paradoja que señala Sommer (167): “Johnny refuses to be contained in Bruno's smug prose. And Bruno strains to be free of Johnny, of the self-doubts and the complexity he inflicts". La tensión entre el personaje narrador y el personaje narrado sigue estando presente, pero no adquiere la dimensión meta-literaria o metaartística que sí posee en Cortázar. Por tanto, las tres relecturas analizadas suponen anular la dimensión del crítico y del papel de la crítica de arte que tanto peso tiene en el original ${ }^{7}$.

La dialéctica entre las dos voces, por muy violenta que sea -y lo es en el caso de Castillo-, apunta ante todo a un escritor que parece desconcertado o frustrado ante la genialidad que surge de un músico 'mediocre' como Griffiths, a la extrañeza ante el contexto en el que se produce y el sujeto en el que se encarna. Esto explica el desprecio que le profesa: un individuo así, parece pensar el narrador sin explicitarlo, no debería tener ese don. "Hay ciertos seres, cierto tipo humano, diría, que tienen la virtud de irritarme, de hacer que pierda el sentido de las proporciones, de los valores. Me llevaría años explicarlo, pero en resumen es esto: los miro y los remiro y me encuentro pensando pero por qué, por qué ellos no. Qué les falta. Y cómo hago yo para descubrirlo" (Castillo 311).

La ausencia de una explicación de esos instantes sublimes que sopla el negro Griffiths, de su genialidad, es lo que explica la reescritura de la voz del narrador que hace Castillo. Esta va en detrimento del cuestionamiento de los límites de la escritura, de la crítica o de su papel frente a la creatividad artística que, como hemos visto, desempeñan un papel protagonista en su hipotexto cortazariano. La voz de los perseguidores, en definitiva, es la misma (véase

\footnotetext{
A propósito del peso que tiene el crítico y la contraposición crítica/creación en "El perseguidor", véase Álvarez Borland (2005), muchas de cuyas observaciones sirven igualmente para contrastar la visión que Castillo, Quiñones o Muñoz Molina desarrollan. Jitrik (1975) fue uno de los primeros en subrayar esta contraposición y en vincularla con la situación del propio Cortázar, que "durante mucho tiempo se pensó a sí mismo como crítico [...], pero que es simultáneamente lo que se conoce como un creador” (Jitrik 83).
} 
el primer apartado). Pero no la del filtro mediante el cual son narrados, que refleja otros intereses y que, en definitiva, apunta en los tres casos de forma inequívoca más hacia el músico y menos hacia un autocuestionamiento del crítico o del escritor.

\section{LA REESCRITURA DEL ESPACIO}

La voz del narrador no es el único elemento reinterpretado en las tres reescrituras de "El perseguidor". Una de las principales vías para actualizar cualquier narración es reubicarla temporal y/o espacialmente. Ninguno de los tres autores en cuestión llega al extremo de Pierre Menard, pero, dados sus nuevos contextos de escritura, cada autor asume igualmente nuevos espacios en los que hacer habitar a sus versiones de Johnny Carter.

Muñoz Molina es el que se mantiene más fiel al original, toda vez que, si bien Billy Swann no vive en París, es igualmente un jazzman estadounidense que lleva una vida nómada por distintas ciudades europeas con la única ambición de beber y tocar. El momento culminante tiene lugar en Lisboa, ciudad que adquiere un marcado estatus simbólico en la novela, cuya configuración como espacio es a grandes rasgos semejante al París cortazariano. Se trata de un centro cultural, un espacio que alberga individuos pertenecientes a todas las clases sociales y que permite mostrar la marginalidad y heterodoxia de los respectivos músicos. Un lugar que no es el hogar de ninguno de ellos y que les permite labrarse su fama y ganarse la vida tocando para un público burgués (empezando por el propio Bruno) sustancialmente irreconciliable con su modo de vida. Es una ciudad que alberga también calles en las que perderse, en las que vagabundear -si bien, en El invierno en Lisboa esto lo hace el protagonista, Santiago Biralbo, no Billy Swann- y en las que ser ingresado en alguna institución que resguarde a los perseguidores de su carácter autodestructivo: sabemos que Johnny ha estado ingresado repetidas veces en centros psiquiátricos e, igualmente, Billy Swann es ingresado durante un significativo periodo de tiempo en una institución regida por monjas (capítulo XIII), a las afueras de la ciudad, para intentar que se recupere de sus excesos con el alcohol.

Por su parte, el relato de Castillo, pero ante todo el de Quiñones, presentan una reelaboración del espacio mucho más sustantiva. Lo relevante en el primer caso es que la vida de Griffiths transcurre en Barracas, suburbio de Buenos Aires, donde interpreta su música en los lugares de más dudosa reputación. 
Del epicentro de las últimas tendencias musicales (París) pasamos a una periferia marginal. Aquí no hay marquesa que haga las veces de mecenas y protectora. Ni hay quien aprecie la genialidad de Griffiths. Solo el narrador parece hacerlo, si bien no se lo manifiesta en ningún momento. Castillo, entonces, adapta la historia conforme a un espacio más realista e inmediato a su contexto de escritura: los suburbios de su ciudad. Este cambio implica un deslizamiento desde la figura puntera del jazz mundial, objeto de veneración y de diversos estudios, al músico anónimo, callejero y ordinario. Su público no es ni la burguesía que acude a escuchar a Billy Swann, ni ningún refinado crítico musical como Bruno. Griffiths, está claro, no graba discos, y el único testimonio de su existencia será el que el propio narrador deja, el propio relato que leemos. En este contexto, New Orleans, que Griffiths evoca con nostalgia y admiración (Castillo 312), hace las veces del París cortazariano, al que Griffiths nunca volverá. Solo mediante los instantes geniales de su música y mediante su interpretación final logrará recuperarse este espacio perdido: "Y Griffiths, en Barracas, se arrodilló como quien habla con Dios y metió su trompeta por un agujero de la empalizada. Y la música y él cayeron del otro lado, en New Orleans. Y se encendieron todos los faroles rojos de las puertas de las muchachas. [...]. Y todas las orquestas de jazz de New Orleans [...] tocaban para ellas" (Castillo 322).

Así concluye el relato, con un momento epifánico en el que Griffiths, en un tugurio de un suburbio bonaerense, logra con su trompeta convocar a todas las orquestas de New Orleans. Castillo tiene interés en subrayar este contraste espacial: ya sabemos dónde se desarrolla la acción, lo cual no quita para que el dato se reitere enfáticamente. La figura del perseguidor, por tanto, se muestra lo bastante versátil como para mantener sus constituyentes fundamentales intactos a pesar de la 'degradación espacial' que experimenta en su traducción al universo narrativo de Abelardo Castillo.

Si la reinterpretación del espacio se mueve aquí en un eje vertical, de lo alto a lo bajo, de la polis cultural a la periferia y al suburbio, Quiñones opta por una traslación que cabe llamar horizontal: no se trata de un espacio dotado de un estatus o de connotaciones más bajas, sino de otro entorno coexistente pero totalmente distinto. De la épica del suburbio pasamos a la épica del gitano andaluz. El nuevo espacio (el Cádiz previo a la Guerra Civil española) implica un perseguidor con rasgos equivalentes a su nuevo contexto: el saxofonista de jazz se transmuta en un gitano cantaor flamenco, lo cual supone, en este nuevo sistema, una posición social y cultural análoga a la del saxofonista de jazz. El vínculo de Miguel Pantalón con la tierra es, en 
realidad, la clave del relato. No ha de olvidarse que el libro al que pertenece este relato (Nos han dejado solos) lleva justamente como subtítulo "Libro de los andaluces”. Pantalón, así, traza un rasgo más del perfil general de su tierra. Es un individuo que, de hecho, "nunca salió de aquí" (Quiñones 468). En esto se diferencia de sus tres colegas, ya que todos ellos son músicos que viven y tocan fuera de su país (Estados Unidos de América en todos los casos menos en este). Miguel Pantalón y su arte, en cambio, son un fruto espontáneo, no cultivado, de su entorno, una muestra en bruto del mismo (al contrario que Falla, al que se presenta como su homólogo culto).

El final del relato, en concordancia con esta idea, subraya el telurismo. Miguel Pantalón se queda dormido en uno de los locales en los que canta tras concluir una de sus intervenciones. Hasta que

...cuando iban a irse, ya de noche, fue cuando se dieron cuenta de que Miguel Pantalón se había muerto.

En una mano, fuerte-fuerte que luego no había quien se la abriera, tenía un puñao de tierra de allí del suelo como el que aprieta el diamante de la India.

Las cosas de él (Quiñones 480).

Se trata de un cierre que, como el del relato de Castillo, subraya el elemento espacial. Si aquel concluye con una reaparición fulgurante de una New Orleans a la que se llega desde Barracas, Quiñones concluye destacando de nuevo el vínculo del protagonista con su tierra. El "puñao" de tierra no es el único elemento que apunta en esta dirección: puede observarse también cómo la sintaxis, la puntuación y el léxico son propios de un registro oral del sur de España. El arte de este perseguidor brota directamente del puñado de tierra al que se aferra mientras canta, haciendo de él un Johnny Carter gitano y flamenco que remite a la metáfora de la tierra como madre y origen. Esto supone una ampliación de la idea original a la vez que una sujeción a sus invariantes, haciendo de la relectura del espacio la principal aportación de Quiñones respecto de su hipotexto.

\section{LA REESCRITURA DEL TIEMPO}

El tiempo es uno de los temas clave de "El perseguidor". La música de Charlie Parker establece una relación idiosincrásica con el tiempo que el protagonista 
trata en varias ocasiones de expresar y que cristaliza en dos de las frases más simbólicas de la obra, que cabe considerar como una suerte de eslogan: "esto lo estoy tocando mañana” y “cómo se puede pensar un cuarto de hora en un minuto y medio?" (Cortázar 135 y 142). La música, al igual que el cronotopo del métro -"viajar en el métro es como estar metido en un reloj" (Cortázar 142), llega a afirmar Johnny-, es para el saxofonista una forma de desautomatizar la percepción de la realidad, una vía para acceder a otra dimensión y experiencia del tiempo. Esto puede enlazarse con un fenómeno más amplio característico de la literatura de Cortázar: el continuo intento de desestabilizar la realidad y nuestra relación y percepción de ella. He aquí el núcleo profundo de la poética cortazariana. A menudo sus personajes tratan de acceder a una "realidad segunda" (Alazraki 141-172) e ingresar así en "el otro lado" (Ortiz 62-63). Evidentemente, el tiempo desempeña un papel protagonista en ello, toda vez que es uno de los aspectos más relevantes de nuestra experiencia del mundo.

Lo que Johnny hace con la música (captar otra visión de la realidad, generar otra vivencia de la dimensión temporal) es lo mismo que pretende hacer el crítico musical que narra la historia a través de lo que escribe y, también, lo que el propio Cortázar intenta a través de muchos de sus relatos. La figura del crítico sirve para plantear no solo la oposición creador/crítico, sino también, en un segundo plano, la oposición música/escritura. Esta polaridad se plantea de distintas maneras a lo largo del texto. Ya desde el principio se explicita que al genial saxofonista "la música [le] saca del tiempo" (Cortázar 137), le hace posible "meter quince minutos en un minuto y medio" (Cortázar 142). Es una idea que se repite de distintas maneras, por ejemplo a través de la metáfora de la maleta en la que no solo cabe un par de zapatos y una camisa, sino que "puedes meter una tienda entera [...], cientos y cientos de trajes, como yo meto la música en el tiempo, cuando estoy tocando, a veces" (Cortázar 139). En cambio, "tú no haces más que contar el tiempo" (Cortázar 132), le reprocha el músico al crítico y escritor, quien, a su vez, se pregunta con cierta frustración “¿por qué no podré hacer como él?” (Cortázar 153155). La contraposición no ya entre las dos figuras y actividades, sino entre los dos medios de expresión, es clara. El escritor y crítico persevera en este tipo de reflexiones cuando nos manifiesta su frustración por no ser capaz de transmitir la belleza que Johnny logra crear. Resuena aquí la concepción del 
crítico como eunuco ${ }^{8}$, como ser castrado para el arte: "no se puede decir nada, inmediatamente lo traduces a tu sucio idioma" (Cortázar 182), le reprocha el saxofonista. Pero, también, la limitación de la escritura para recrear ese tipo de experiencia estética que surge del saxo del músico.

El veredicto de Johnny sobre el libro que acaba de publicar el narrador parece ser favorable, pero "faltan cosas" (Cortázar 191). Se subraya, de nuevo, la imposibilidad de lograr el efecto de extrañamiento espacio-temporal que propone Johnny. Johnny, el perseguidor, persigue esta percepción particular y estética del tiempo y el espacio. El crítico que narra la historia persigue captar y trasladar esta misma percepción espacio-temporal. Y, en último término, ¿qué persigue "El perseguidor"? También (re)crear este mismo efecto.

Queda de este modo esbozado que "el problema de la música en 'El perseguidor' es el problema del tiempo" (González Riquelme 2003), algo que el título mismo ya simboliza y que permea todos los niveles de la narración. Y esta misma lectura es la que reitera Muñoz Molina y la que refleja a través de su Billy Swann. El propio autor español ha reflexionado sobre el asunto a través de diversos artículos, normalmente publicados en prensa. En "Cortázar músico", por ejemplo, analiza el estereotipo literario que Cortázar crea en su relato, señalando que el parecido con el músico de carne y hueso, Charlie Parker, es en realidad muy superficial "y tiene más que ver con un cierto estereotipo sobre el músico de jazz como una variante del artista maldito que con la realidad de la vida de Charlie Parker, o casi de cualquier músico de esa generación y esa escuela" (Muñoz Molina 2015). Por esto mismo, creo yo, tiene poco sentido identificar la figura de Billy Swann con músicos reales, ya sea de nuevo Charlie Parker u otros como Chet Baker (Sperber 50). El argumento que aquí se sostiene, por tanto, es que Billy Swann es una nueva elaboración de un tipo literario que el propio Muñoz Molina reconoce como tal. Y aquí, como me propongo mostrar, no está ausente un componente tan relevante en su fuente como lo es la dimensión temporal.

Tal vez el aspecto más evidente en el que esto se manifiesta sea el momento culminante de la novela, en su penúltimo capítulo (capítulo XIX), cuando

Empleo esta noción como metáfora que sintetiza la idea del crítico literario, musical o artístico como un individuo que se caracteriza por su incapacidad creadora, y que evalúa obras artísticas sin tener la posibilidad de producirlas él mismo. El término "eunuco" lo ha empleado recientemente con este mismo valor Andrés Neuman (27) en uno de sus "barbarismos". 
Biralbo y Billy ofrecen el que será el último concierto del segundo. Como ya se ha dicho, el concierto ocupa el capítulo en su integridad y se presenta a través de minuciosas y evocadoras descripciones de la música interpretada. Ya próximos al momento final y culminante del mismo, comienza a percibirse con claridad la alteración de la experiencia del tiempo que implica la música que los protagonistas crean. Ellos establecen "con su música una línea inaudible que lo circundaba como una campana de cristal, un tiempo únicamente suyo en el interior del tiempo disciplinado por otros" (Muñoz Molina 181). Es justamente el "tiempo subjetivo, ajeno al reloj" que Galindo Ulloa (336) analiza respecto de "El perseguidor" el que entra en juego aquí. La música genera una especie de "campana de cristal" que permite una vivencia alternativa del tiempo, lo cual vuelve a subrayarse unas líneas después: "entonces Billy Swann se quitó la trompeta de la boca y Biralbo pensó que habían pasado varias horas y que el concierto iba a terminarse, pero nadie aplaudió, no se oyó ni un rumor en la sobrecogida oscuridad donde la última nota aguda de la trompeta no se había extinguido aún" (Muñoz Molina 181). Billy Swann, en efecto, ha conseguido meter "varias horas" en los pocos minutos que ha estado tocando.

La persecución de esta experiencia temporal alternativa se traslada a quienes están acompañando a Billy Swann. Muñoz Molina emplea de hecho este mismo término clave ("persecución") para definir los esfuerzos de los músicos en escena: "cuando Biralbo alzaba los ojos del piano veía su perfil rojizo y contraído y sus párpados apretados como una doble cicatriz. Ya no podían seguirlo y se dispersaban, cada uno de los tres afanosamente extraviado en su persecución" (Muñoz Molina 181). Más allá de la intertextualidad que implica el empleo de esta última palabra, la idea de fondo es exactamente la misma: como Johnny Carter, Swann y sus músicos persiguen una desautomatización de su percepción del tiempo, un extrañamiento ante la magnitud temporal de la realidad que solo parece poder perseguirse y aprehenderse mediante la música. Plenamente consecuente con la relectura del relato de Cortázar que se plantea, el momento final y culminante del concierto y del capítulo lo constituye la experiencia subjetiva del tiempo que experimenta Biralbo (el protagonista de la novela), que parece revivir los hechos fundamentales de los últimos años de su vida y de su relación con Lucrecia durante la intervención de su amigo, hasta que "como si despertara se dio cuenta de que ya no oía la voz de Billy Swann” (Muñoz Molina 182).

De esto se sigue que El invierno en Lisboa sitúa en su escena culminante la idea de temporalidad heredada de "El perseguidor". Pero no se limita a ello, 
sino que también desarrolla una elaborada red de niveles temporales del relato que cabe relacionar con ello. Uno de los rasgos formales más llamativos de la novela de Muñoz Molina es la relativamente compleja estructura temporal sobre la que se construye, estructura además que en ningún momento va en detrimento de la fluidez y claridad narrativas. Si Johnny, cuando toca, mete horas en unos pocos minutos, el narrador, cuando narra, mete la historia de varios años en las pocas horas que pasa con Santiago Biralbo. Esto es posible gracias a que la historia nos llega a través de lo que Biralbo le cuenta años después al narrador, que a su vez narra los hechos con posterioridad a este encuentro9.

Es cierto que, en los relatos de Quiñones y Castillo, la narración de las interpretaciones musicales de los protagonistas implica, de algún modo, una elongación del tiempo, que parece dilatarse ante la experiencia estética que el narrador vive con la música de sendos perseguidores. No obstante, ha quedado de manifiesto que, en el caso de Muñoz Molina, este aspecto adquiere mucha mayor relevancia, hasta el punto de que puede hablarse de una amplificatio del concepto del tiempo de "El perseguidor". Esto sucede no tanto mediante el diálogo directo con el músico en cuestión, sino a través de la descripción de su música, así como, de modo más indirecto, a través de la configuración de los niveles temporales del relato.

\section{CONCLUSIONES}

Toda cita, variación y reescritura implican una interpretación. Este artículo identifica tres interpretaciones de "El perseguidor" ("Noche para el negro Griffiths", de Abelardo Castillo; "El testigo", de Fernando Quiñones; y el personaje de Billy Swann en El invierno en Lisboa, de Antonio Muñoz Molina) y las contrasta con su origen. ¿Qué consecuencias se extraen entonces de ello? En primer lugar, cabe argumentar que el perseguidor -y no Johnny Carter en tanto que trasunto ficcional de Charlie Parker- se ha convertido en un mito contemporáneo, si por esto entendemos una figura simbólica que condensa una actitud ante el arte y el mundo y que reaparece siempre

9 La construcción temporal de El invierno en Lisboa, así como el papel que el tiempo desarrolla en la novela y cómo se relaciona con el jazz, pueden desarrollarse mucho más. Para ello, véase Pérez Lasheras (1994) y Franz (2000). 
con una serie de rasgos invariables, los cuales suponen a su vez la narración de una historia - de su historia- con aspectos igualmente comunes. Los tres textos elegidos permiten perfilar las invariantes más idiosincrásicas que constituyen al perseguidor, cuyos rasgos van mucho más allá de los de un jazzman cualquiera. La identidad del mito, por otro lado, no está tanto en sí mismo, en la historia que nos cuenta la fuente original, cuanto en la lectura de ella que se hace con posterioridad, en los rasgos que se perpetúan al modo darwiniano y, en virtud de ello, adquieren valor identificativo. En este caso, se ha argumentado que entre ellos se cuentan 1) el don para la música, es decir, la genialidad; 2) la ausencia de educación musical formal, que explica la elección de géneros como el jazz o el flamenco; 3) la marginalidad, que alude a una posición social y económica bajas y en la que suele estar presente la adicción a las drogas; 4) la inconsciencia o insatisfacción ante las propias dotes musicales; 5) la 'frase intuitiva', que captura lo escurridizo de la música y de la visión que de ella tienen los perseguidores; 6) la extravagancia cercana a la locura, que enlaza con el tópico cultural del genio; 7) el desinterés por el mundo y por las cuestiones mundanas como el dinero o el éxito; 8) la inadaptación a las normas y convenciones sociales; 9) la muerte prematura (con la excepción ya explicada del negro Griffiths) y 10) el ángel protector femenino que lo acompaña.

Asimismo, pueden fijarse otros rasgos que sobrepasan la caracterización del personaje, como 11) la 'mediación' de su figura a través de (al menos) otra voz, que narra la historia, o 12) el tiempo, su percepción alterada y alternativa como tema clave del relato. La fijación de estas invariantes, además, constituye un argumento de peso en contra de determinadas interpretaciones posibles de estas obras. Por ejemplo, la de ver a Griffiths como un simple músico mediocre, sin mayor matización, basándose en que el narrador de la historia así lo afirma, o el debate en torno a si Billy Swann se inspira más en Charlie Parker, Chet Baker o en cualquier otra figura histórica.

Ahora bien, todo esto no implica que los textos analizados sean una mera copia del original. Se ha mostrado que, lejos de ello, implican una relectura que pivota en torno a tres ejes: la reescritura de la voz, del espacio y del tiempo. Cada una de las narraciones potencia con especial fuerza uno de estos aspectos. Así, el relato de Castillo funciona ante todo como una reescritura de la voz, ya que pone en primer plano la marginalidad y 'mediocridad' vital del perseguidor, y lo hace a través de una voz mediadora que también adquiere notoriedad. La narración de Quiñones se construye a partir de la reescritura del espacio, ya que traslada la acción a Andalucía y subraya con diversos 
recursos el telurismo del personaje principal. La novela de Muñoz Molina, finalmente, actúa como una reescritura del tiempo, toda vez que explora la dimensión temporal y la percepción desautomatizada de ella heredada de Cortázar.

Es factible, además, que nazcan o aparezcan más perseguidores. Este artículo permite entenderlos como eslabones de la cadena literaria a la que pertenecen y dialogar con la tradición crítica y bibliográfica sobre Cortázar, ya muy frondosa, desde un ángulo poco frecuentado: el de la reescritura directa y la aportación crítica que ello supone. Obras que aquí han quedado al margen como Siberia blues, de Néstor Sánchez, o algunos cuentos de Hipólito G. Navarro, permitirían ampliar este análisis, no ya en tanto que nuevos ejemplos del mismo fenómeno, sino como textos influidos por "El perseguidor" que, no obstante, no pueden considerarse como reescritura o adaptación del mismo. Sería interesante ampliar este análisis y comprobar qué implican otros tipos de influencia menos explícitos. Este artículo, entonces, pretende dar algunas claves para ello.

En definitiva, este análisis muestra que los hermanos menores de Johnny permiten establecer un perfil literario constante. El interés radica en que no se trata de interpretaciones críticas, vehiculadas mediante ensayos o artículos, sino de interpretaciones literarias, que han de equilibrar la dialéctica entre la repetición y el cambio que toda reescritura supone. El número de rasgos que se puede alterar es limitado si se quiere que el producto final se perciba como remake del original; a la vez, cada autor ha de introducir ciertas variaciones o cambios que den cuerpo a su propia visión del relato. Detectar y analizar en qué términos se da este equilibrio es también un modo de aportar nuevas interpretaciones de la obra de Cortázar.

\section{BIBLIOGRAFÍA}

Alazraki, Jaime. Hacia Cortázar: aproximaciones a su obra. Barcelona: Antrophos, 1994.

Álvarez Borland, Isabel. "Cortázar: On Critics and Interpretation”. Julio Cortázar. Bloom 's Modern Critical Views. Ed. Harold Bloom. Philadelphia: Chelsea House Publishers, 2005. 183-194.

Aquino, Andrea y Alejandro Pignataro. "Espacios ficcionales: la ambigüedad de los límites en las narraciones fantásticas. Acercamiento a 'La caricia más profunda' (Julio Cortázar) y 'Mis vecinos golpean' (Abelardo Castillo)”. Tenso Diagonal 01 (2016): 102-112. 
Borrello, Rodolfo. "Charlie Parker: «El perseguidor»", Cuadernos Hispano-americanos 364-366 (1980): 573-594.

Castillo, Abelardo. Cuentos completos. Los mundos reales. Buenos Aires: Alfaguara, 1997. Diarios 1954-1991. Buenos Aires: Alfaguara, 2014.

Cortázar, Julio. Las armas secretas. Ed. Susana Jakfalvi. Madrid: Cátedra, 1987.

Franz, Thomas. R. "Jazz Specifics and the Characterization and Structure of El invierno en Lisboa". Revista de Estudios Hispánicos 34.1 (2000): 157-164.

Galindo Ulloa, Javier. "El tiempo, el sueño y la música en 'El perseguidor'”. Julio Cortázar: perspectivas críticas. Ensayos inéditos. Coords. Popovic Karic, Pol y Fidel Chávez Pérez. Monterrey, México: Miguel Ángel Porrúa, 2012. 317-343.

Genette, Gérard. Palimpsestos. La literatura en segundo grado. Madrid: Taurus, 1989.

González Riquelme, Andrés. "La máquina musical de 'El perseguidor' de Julio Cortázar", Acta Literaria 28 (2003): 33-44.

Jitrik, Noé. “Crítica satélite y trabajo crítico en «El perseguidor» de Julio Cortázar”, en Producción literaria y producción social. Buenos Aires: Sudamericana (1975): 82-129.

Menczell, Gabriella. Incipit y subtexto en los cuentos de Julio Cortázar y Abelardo Castillo. Budapest: Akadémiai Kiadó, 2002.

Morello-Frosch, Marta. "Prólogo", en Castillo, Abelardo. Cuentos Completos. Los mundos reales. Buenos Aires: Alfaguara (1997): 11-26.

Muñoz Molina, Antonio. El invierno en Lisboa. Barcelona: Seix Barral, 1987.

“Cortázar músico” El País. 7 dic. 2013. Web: Elpais.com 7 sept. 2015.

Neuman, Andrés. Barbarismos. Madrid: Páginas de Espuma, 2014.

Ortiz, Carmen. Julio Cortázar. Una estética de la búsqueda. Buenos Aires: Editorial Almagesto, 1994.

Paredes, Alberto. Abismos de papel: los cuentos de Julio Cortázar. México D.F.: Universidad Nacional Autónoma de México, 1988.

Pérez Lasheras, Antonio. "Tiempo real/ Tiempo narrativo en El Invierno en Lisboa de Antonio Martínez Molina". Actas del congreso en homenaje a Rosa Chacel: ponencias y comunicaciones, María Pilar Martínez Latre (coord.). Logroño: Universidad de Logroño (1994): 223-228.

Quiñones, Fernando. Tusitala. Cuentos completos. Madrid: Páginas de Espuma, 2003.

Sommer, Doris. "Grammar Trouble: Cortazar's Critique of Competence”. Julio Cortázar. Bloom's Modern Critical Views. Ed. Harold Bloom. Philadelphia: Chelsea House Publishers (2005): 149-182.

Sperber, Richard. The Discourse of Flanerie in Antonio Munoz Molina's Texts. Lewisburg: Bucknell University Press, 2015. 